\title{
ST-Segment Elevation and Normokalemia in Acute Diabetic Ketoacidosis: Case Report and Brief Literature Review
}

\author{
S. Bryn Dhir,' Abbas Husain. ${ }^{2}$
}

\begin{abstract}
Background: Diabetic Ketoacidosis (DKA) is a life-threatening complication of Diabetes Mellitus Type 1 (DM1) and requires prompt management; however, benign transient electrocardiographic (ECC) abnormalities with normal serum potassium levels can be seen in diabetic patients secondary to metabolic changes. Understanding the varying presentation among patients provides valuable insight into the management of this seemingly uncommon and benign diagnosis. The Case: A 24-year-old male with a history of DM1 presented to the Emergency Department (ED) with ST-segment elevation, normal potassium levels and metabolic acidosis. The patient was found to be in DKA with benign cardiac manifestations. Conclusion: The correction of underlying metabolic abnormalities in DKA and the awareness of the benign cardiac pseudo pathology on ECG allows for effective management and personalized patient care.
\end{abstract}

Key Words: Diabetic ketoacidosis; Diabetes mellitus type 1; Myocardial infarction; Pseudo pericarditis; Normokalemia (Source: MeSH-NLM).

\begin{abstract}
Introduction
Patients with DM are at risk for life-threatening complications such as cerebral edema, noncardiogenic pulmonary edema, DKA, and a subset of complications secondary to metabolic imbalances. Among the most concerning, DKA requires rapid diagnosis and is often associated with viral illness, infections, compliance of insulin therapy, stroke, trauma, surgery, alcohol abuse, pregnancy and severe dehydration. DKA is responsible for acute metabolic complications with fluid-electrolyte and acid-base abnormalities such as increased potassium levels, errors in metabolizing fat, carbohydrates and protein, and ECG abnormalities. However, the presentation of DKA varies greatly between patients and is often unrecognized or misdiagnosed.' To the best of our knowledge, this is the first case report to highlight the clinical presentation and management in a young male patient with DM1 in DKA with normal serum potassium levels and ST-segment myocardial infraction (STEMI) in the Emergency Department (ED) and Intensive Care Unit (ICU) at the Staten Island University Hospital, Staten Island, New York, USA.
\end{abstract}

\section{The Case}

A 24-year-old male with a history of insulin-dependent DM1 presented to the ED with abdominal pain, nausea, and fifteen episodes of nonbloody, non-bilious vomitus for 24 hours. Admission vital signs evidenced tachycardia with no other abnormalities: right arm blood pressure 118/82 mmHg; pulse rate 102; respiratory rate 18; oxygen saturation on room air $97 \%$; oral temperature $35.8^{\circ} \mathrm{C}$; auscultation of the heart and lung fields revealed normal S1, S2, rate and rhythm; bilateral breath sounds without rales, rhonchi or wheezes. The patient was awake and oriented to person, place, time, situation and spoke coherently. Associated symptoms included: diffuse, intermittent, cramping epigastric pain without fever or chills. On exam, his abdomen was soft, non-tender, without guarding or peritoneal signs, and positive for bowel sounds in all four quadrants. He denied recent travel, tobacco or illicit drug use and indicated regular compliance of his insulin pump with the exception of the previous day. On further evaluation, he indicated consumption of approximately two to three beers and two to three cups of hard liquor the previous day at a party. Initial and repeated 12 lead ECG showed a STEMI (Figure 1) and an anteriorposterior chest $\mathrm{x}$-ray was unremarkable for evidence of acute cardiopulmonary disease (Figure 2). Laboratory work up of cell counts, metabolic and urine profile, and cardiac biomarkers were investigated and significant results were noted for acetone, serum glucose, urine glucose and ketones (Table 1).

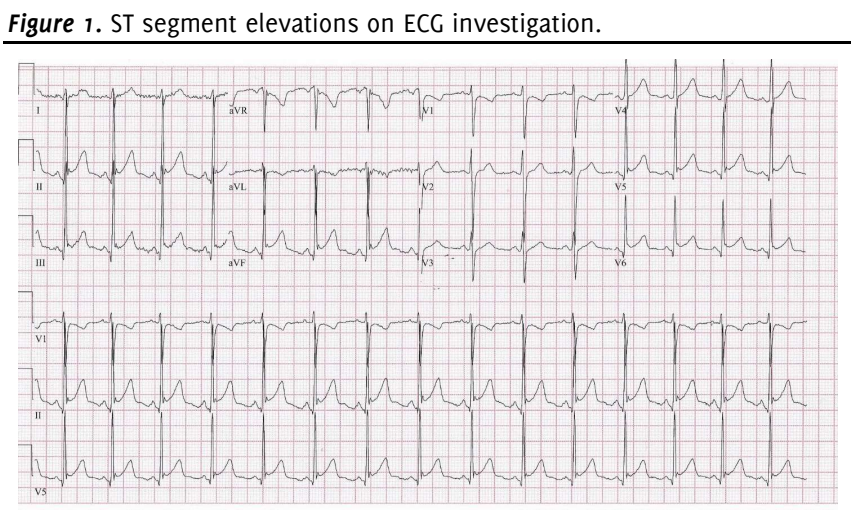

The patient was diagnosed to be in DKA with acute kidney injury (AKI). Management with $0.9 \%$ sodium chloride, potassium chloride, $20 \mathrm{mg}$ famotidine, 4mg ondansetron hydrochloride, and continuous insulin infusion was initiated in the ED. A third ECG continued to showed pathology for a STEMI. Upon consultation with a cardiologist, percutaneous coronary intervention was not indicated at the time. The care plan included optimizing medical management with adjustments to subcutaneous (SC) insulin, and inpatient care in the ICU under critical care. The total time in the ED was approximately 2.5 hours.

Approximately eight hours after the patient presented to the ED, the cardiac manifestations resolved and the AKI secondary to mild dehydration showed improvement. The patient was comfortably ambulating and tolerating food orally (approximately 20 and 24 hours later). Repeat laboratory work-up at 20 hours and 46 hours showed

. HBA, Medical Student, International American University College of Medicine, Plano, Texas, USA.

, MD FACEP, Staten Island University Hospital, Emergency Department, Staten Island, NY, USA.

About the Author: Bryn Dhir is a medical student committed to emergency medicine, wilderness medicine and disaster management. Bryn is the recipient of the medical student awards for excellence in emergency medicine from the Society for Academic Emergency Medicine, and the American College of Emergency Physicians. Bryn's research interests include neuroscience, proteomics, climate change, and is dedicated to medical education and leadership skill development.

Correspondence:

Bryn Dhir

Address: 1255 W 15th Street, Suite 100, Plano, Texas 75075 USA

Email: bryndhir@icloud.com
Editor: Mihnea-Alexandru Găman Student Editor: Mohamed Gad Submission: $\operatorname{Sep} 18,2019$ Acceptance: Mar 02, 2020 Publication: Apr 30, 2020 Process: Peer-reviewed 
anion gap and metabolic improvements. Upon detailed review of the case and medical education, the patient was discharged approximately 48 hours after ICU admittance.

Figure 2. Anterior-Posterior Chest X-Ray.

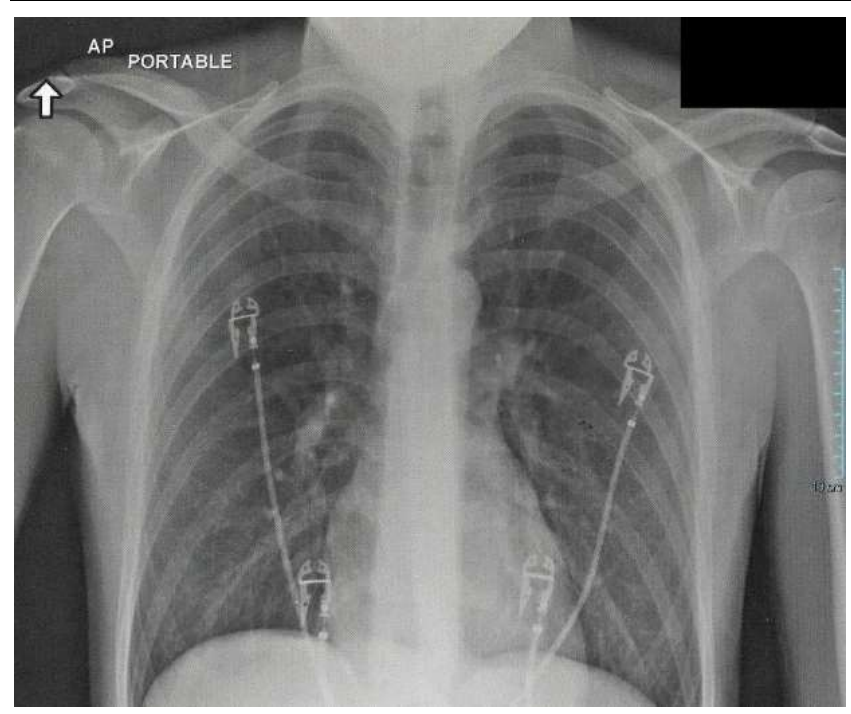

Table 1. Laboratory Investigation and Results: Basic Metabolic Profile.

\begin{tabular}{lccc}
\hline Basic Metabolic Profile & o hrs & 20 hrs & 46 hrs \\
Anion Cap $(\mathrm{mmol} / \mathrm{L})$ & 23 & 12 & 11 \\
Clucose $(\mathrm{mg} / \mathrm{dL})$ & 441 & 163 & 231 \\
Blood Urea Nitrogen $(\mathrm{mg} / \mathrm{dL})$ & 28 & 14 & 10 \\
Creatinine $(\mathrm{mg} / \mathrm{dL})$ & 1.75 & 0.91 & 0.75 \\
BUN:Cr Ratio & 16 & 0.154 & 13.3 \\
GFR & 48 & 102 & 128 \\
Sodium $(\mathrm{mmol} / \mathrm{L})$ & 133 & 135 & 137 \\
Potassium $(\mathrm{mmol} / \mathrm{L})$ & 4.2 & 3.9 & 3.7 \\
Chloride $(\mathrm{mmol} / \mathrm{L})$ & 97 & 1.4 & 99 \\
Calcium $(\mathrm{mg} / \mathrm{dL})$ & 10.5 & 9.1 & 9.1 \\
Co2 $(\mathrm{mmol} / \mathrm{L})$ & 13 & 19 & 27 \\
Lactic Acid $(\mathrm{mmol} / \mathrm{L})$ & 2.7 & & \\
\hline
\end{tabular}

Legend: GFR - Glomerular Filtration Rate, BUN:Cr - Blood Urea Nitrogen to Creatinine ratio.

\section{Discussion}

DKA is a life-threatening complication of DM and requires rapid diagnosis by medical care teams involved in patient care, and closedloop communication aids favorable outcomes. The understanding of the vastly different patient presentations can allow for cost-effective and appropriate personalized care, as was seen in this patient case. Medical management of DKA includes correction of electrolyte abnormalities, in particular potassium and phosphate; fluid replacement; as well as the administration of bicarbonate, though controversial. Recommendations for insulin therapy guided by serum potassium indicate that low-dose IV insulin should be used if the serum potassium is greater than or equal to $3.3 \mathrm{mEq} / \mathrm{L}$, whereas therapy should be delayed if the level is less than $3.3 \mathrm{mEq} / \mathrm{L}$ in order to allow for potassium replacement. Imaging and investigations are often guided by patient presentation as well as hospital and physician practices, and can range from conservative to invasive with costly measures such as coronary angiography imaging.
As outlined in this case, the transition of insulin therapy from IV to SC in the ICU is a widely adopted practice by hospitals managing DKA, and various algorithms and criteria exist for the safe transition. ${ }^{2}$ Other treatment options, such as the use of IV heparin in severe cases of DKA for the prevention of pancreatic complications in patients with hypertriglyceridemia, was not indicated for this case. In addition to evidence-based medicine and applying clinical research literature to decipher uncommon disease presentations, the understanding of ECG abnormalities and insulin compliance allows for appropriate patient care with favorable and uneventful outcomes. Furthermore, although serum potassium levels may be normal or elevated in DKA, a total body potassium deficit may exist. This means understanding the pathophysiology of the cellular movement of potassium can eliminate further decreased levels caused by rehydration dilution, urinary loss, acidosis correction, or reentry into cells. Metabolic changes are often reversible with rehydration as well as the correction of the underlying abnormalities, and ECG can be a useful tool to guide potassium replacement. ${ }^{3}$ Other factors influencing benign transient ECG changes in DKA include idiopathic pericarditis, nonspecific cardiac enzymes abnormalities, vasospasms, autoimmune diseases and viruses as metabolic acidosis and Brugada syndrome do not solely contribute to ST-segment changes. ${ }^{4}$

Despite the widely accepted and common practices of medical management for the disease, the diagnosis is often unrecognized or mistaken for another infection (alcohol abuse, drug use, lactic acidosis, STEMI) and the presentation of DKA vastly differs between patients. The awareness among physicians, health care workers and the scientific community is lacking and remains ambiguous. Novel insight into DKA and DM was first provided by seminal cases published in the medical literature by Campbell et al., 1977, and identified the complications associated with uncontrolled diabetes. The abnormal metabolic states were attributed to the severe changes associated with water and electrolyte imbalances, and manifested as subepicardial injury presenting as pseudo pericarditis with ECG abnormalities such as the displacement of the J point and ST-segment elevation without a QRS complex. ${ }^{5}$ In addition, the effects of DKA and hyperkalemia were observed in the patients from the Campbell and colleagues (insert year article was published here) study, in which dry pleurisy was associated with pleural pain and friction rub. ${ }^{6}$ Furthermore, idiopathic causes of benign acute pericarditis in diabetic patients with hyperkalemia and pseudo-anterior wall infarction are also described in early cases reported by Bennett and Blake (1971).7 Although our patient presented with a normal serum potassium level, compared to patient cases described above, this case report is similar in that pathology attributed to infectious causes were ruled out early because of lacking clinical signs and symptoms (friction rubs, chest pain, pericardial effusion, fever, changes to white blood cell counts).

Case reports such as this highlight the differences and similarities in the presentation of signs and symptoms of a young patient in DKA. The young patient in DKA with seemingly alarming ECG abnormalities and normal potassium levels contrasts with adult male patients that have Type 2 DM, noncompliant with oral insulin therapy and may present with acute inferior pseudo infarction, normal potassium levels, and extended hematemesis. ${ }^{8}$ Further, noncompliant teenage males with normokalemia can present with T wave inversion in leads II, III, aVf, V4 6. ${ }^{9}$ Hyperkalemia in male patients with $\mathrm{DM}_{1}$ in the same age range as our patient is also possible, and ECG can show ST-segment elevation in leads I, II, aVL, aVF, V2-V6, as well as ST-segment depressions in lead aVR. ${ }^{10}$ Although the presentation of patients with DM in DKA with ECC abnormalities is often unrecognized or misdiagnosed, understanding the cardiac manifestation mimicking STEMI and correcting metabolic abnormalities is essential in providing rapid, cost-effective personalized care to patients. 


\section{References}

1. Hirsch IB, Emmett M. Diabetic ketoacidosis and hyperosmolar hyperglycemic state in adults: Treatment. In DM Nathan, JE Mulder (Eds.), UpToDate;2020 [cited 22 Feb 2020]. Available from: https://www.uptodate.com.

2. Perilli G, Saraceni C, Daniels MN, Ahmad A. Diabetic Ketoacidosis: A Review and Update. Curr Emerg Hosp Med Rep. 2013 Dec 22;1:10-17.

3. Chava NR. ECG in Diabetic Ketoacidosis. Arch Intern Med. 1984 Dec;144(12):23792380.

4. Stewart JS. Management of cardiac arrest with special reference to metabolic acidosis. Br Med J. 1964 Feb 22;1(5381):476-479.

5. Campbell LW, Duncan LJ, Clarke BF. Pericarditis in diabetic ketoacidosis. Br Heart J. 1977 Jan;39(1):110-112.

6. Armanino LP, Ory EM. Acute pleurisy as a dehydration phenomenon in diabetic precoma. Am J Med Sci. 1946 May;211:597-601.
7. Bennett KR, Blake TM. (1971). Pseudopericarditis in diabetic ketoacidosis. South Med J. 1971 May;64(5):610-2.

8. Aksakal E, Ulus T, Bayram, Bayram E, Duman H. Acute inferior pseudoinfarction pattern in a patient with normokalemia and diabetic ketoacidosis. Am J Emerg Med. 2009 Jan 1;27(2):251-251.

9. Kale T, Agrawal H, Pandit $\mathrm{R}$, Naheed $\mathrm{Z}$. T Wave investigation in diabetic ketoacidosis with normokalemia in an adolescent. Pediatr Cardiol. 2013 August;34(6):1508.

10. Fronczyk A, Moleda P, Majkowska L. Clinical and ECG patterns of pseudoinfarction in a young man with type 1 diabetes, diabetic ketoacidosis and normokalaemia. Clin Dibet. 2016 July;5(2):73-76.

\section{Acknowledgments}

The authors would like to acknowledge Emily Bokser, MD for critical review.

Conflict of Interest Statement at Funding

The Authors have no funding, financial relationships or conflicts of interest to disclose.

Author Contributions

Conceptualization, Investigation, Visualization, and Writing - Original Draft: SBD. Writing - Review $九$ Editing: SBD and AH.

Cite as:

Dhir SB, Husain A. ST-Segment Elevation and Normokalemia in Acute Diabetic Ketoacidosis: Case Report and Brief Literature Review. Int J Med Students. 2020 Jan-Apr;8(1):33-35.

This work is licensed under a Creative Commons Attribution 4.0 International License

ISSN 2076-6327

This journal is published by the University Library System, University of Pittsburgh as part of the Digital Publishing Program and is co-sponsored by the University of Pittsburgh Press. 